\title{
ALGORITHM FOR DETERMINING THE DIFFERENCE PARAMETERS OF VARIOUS NON-PERIODIC SIGNALS
}

\author{
Zaal Azmaiparashvili, Prof. Head of the Department of Microprocessor and Measuring Systems, \\ Faculty of Informatics and Control Systems, Georgian Technical University, Georgia \\ Nona Otkhozoria, Prof. Department of Microprocessor and Measuring Systems, Faculty of \\ Informatics and Control Systems, Georgian Technical University, Georgia
}

DOI: https://doi.org/10.31435/rsglobal_conf/30052021/7572

\begin{abstract}
The article proposes an algorithm for finding the characteristic parameter, which makes it possible to distinguish various non-periodic signals from each other and to obtain a quantitative estimate of the observed processes. The tables and graphs of the initial data, mathematical expressions for finding the characteristic parameter and the algorithm for processing the investigated signals.
\end{abstract}

Keywords: Non-periodic signals, Experiments, Statistical features, characteristic parameter

Introduction. In recent years a wireless electricity transmission becomes topical.

Several wireless power transmission devices has been created and for one of them a patent was received (Georgia Patent No. P 6089, 2012).

During experiments on wireless power transmission, we are faced with the need of studding and obtaining information about the processes occurring during electricity transmission namely, the process of accumulation and releasing energy to generate power output of pulses at which high voltage in the range of tens of kilovolts is generated. These devices must meet the following requirements: high energy density; high electric strength; the ability to provide high current discharge; the duration of the energy storage (small leak rate); high efficiency during charging and discharging; high power multiplication factor (the ratio of the discharge to the charge power); ability to work with repetition pulses; durability, etc. (Bluhm, 2006).

Obviously, some of these requirements contradict each other, and depending on the field of application and the particular restriction, it is necessary to look for certain compromises. We turned to indirect acoustic research methods. To control these processes, we used a piezoelectric acoustic transducer, which allowed to observe the process of accumulation and release of energy, by measuring certain parameters of acoustic signals that high-voltage discharge radiates. By analyzing the information obtained, we can judge about the taking place process, for example, count the number of generated high-power pulses in a certain period of time and estimate the power of the energy radiation, contributing to evaluate the efficiency of the device

\section{Working Methodology.}

To perform the above experiments a microprocessor device that provides a digital processing of investigated low frequency signal was used.

The microcontroller includes an analog-to-digital converter, with which the analog signal is converted to a digital form. Those. It takes place digitization of the test signal over time at a constant pitch sampling - $\Delta \mathrm{t}$. Conversion results in a digital (binary) code are written to the internal flash memory of the microcontroller (Lyons, 2010) and the data are evaluated. Some statistical parameters of the test process are, - calculating the minimum and maximum values of a series of observations, the range, amplitude, mean value, deviation from the mean.

The results of the experiment are given in Tables 1 and 2 . 
Table 1.

\begin{tabular}{|c|c|c|c|c|}
\hline \multicolumn{5}{|c|}{ Charging - series of observations } \\
\hline N & Series 1 & Series 2 & Series 3 & Series 4 \\
\hline 1 & 255 & 145 & 255 & 206 \\
\hline 2 & 150 & 83 & 196 & 135 \\
\hline 3 & 144 & 255 & 243 & 142 \\
\hline 4 & 216 & 119 & 100 & 220 \\
\hline 5 & 101 & 129 & 197 & 121 \\
\hline 6 & 183 & 77 & 142 & 211 \\
\hline 7 & 179 & 211 & 155 & 174 \\
\hline$\cdot$ & & & & \\
\hline 63 & 124 & 137 & 124 & 124 \\
\hline 64 & 126 & 127 & 123 & 124 \\
\hline
\end{tabular}

Table 2.

\begin{tabular}{|c|c|c|c|c|c|}
\hline \multicolumn{7}{|c|}{ Discharging - series of observations } \\
\hline N & Series 1 & Series 2 & Series 3 & Series 4 & Series 5 \\
\hline 1 & 138 & 138 & 98 & 115 & 149 \\
\hline 2 & 141 & 141 & 168 & 120 & 144 \\
\hline 3 & 137 & 137 & 111 & 136 & 130 \\
\hline 4 & 140 & 140 & 123 & 158 & 137 \\
\hline 5 & 128 & 128 & 209 & 133 & 148 \\
\hline 6 & 135 & 135 & 115 & 134 & 127 \\
\hline 7 & 122 & 122 & 134 & 135 & 134 \\
\hline$\cdot$ & & & & & \\
\hline 63 & 127 & 125 & 124 & 126 & 127 \\
\hline 64 & 127 & 126 & 125 & 126 & 127 \\
\hline
\end{tabular}

Below are given graphs of non-periodic signals (Fig. 1,2).

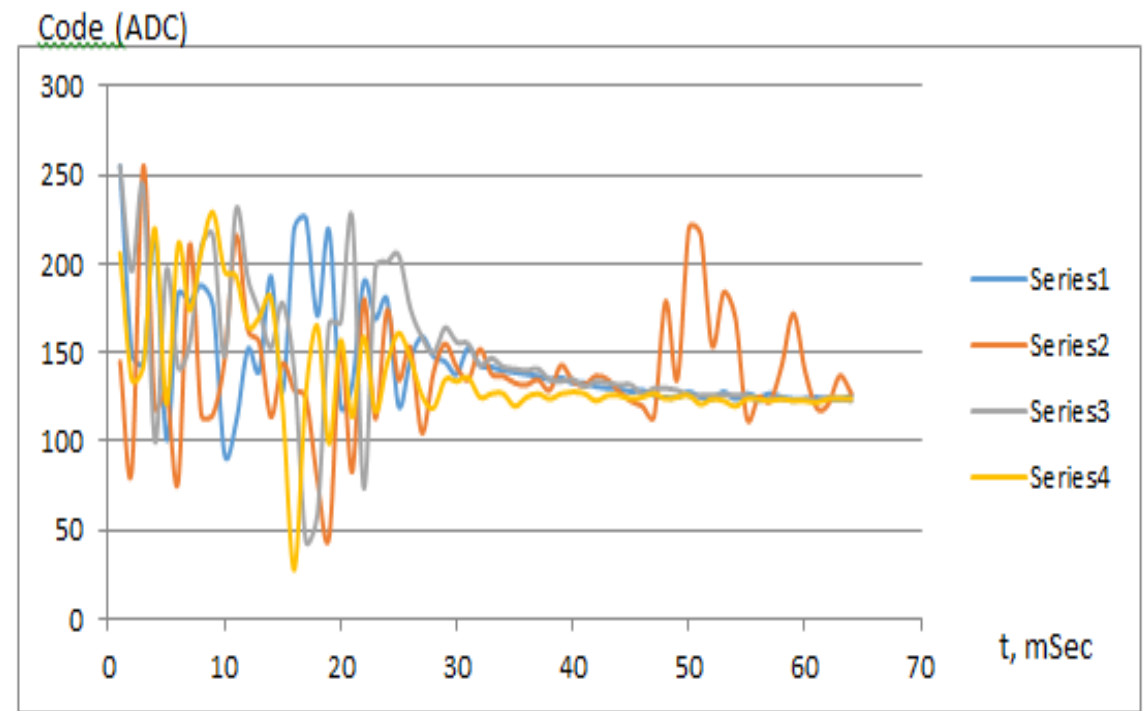

Fig. 1. series of observations of the first process

Our aim is to develop criteria for evaluating the differences between the two varieties of processes, allowing to identify and to quantify the observed processes.

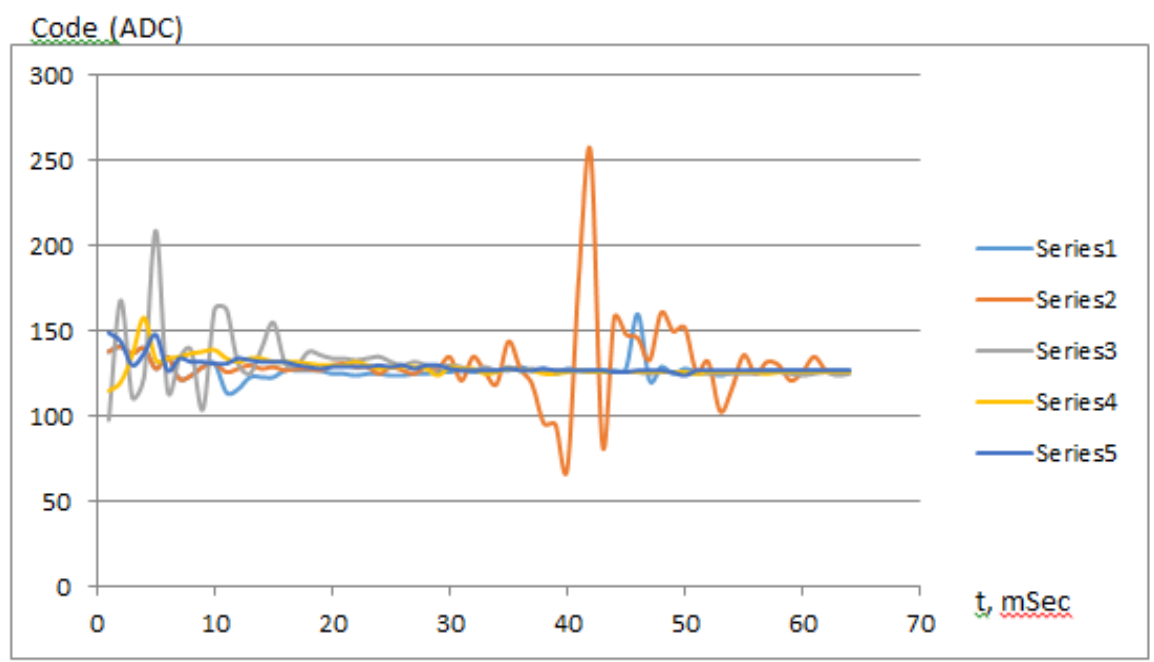

Fig. 2. shows series of observations of the second process 
In statistics, there are different algorithms homogeneity criteria for the two observed groups. To verify these groups Fisher test was used. According to this criterion could not be established the uniformity of the group, as the average values and the corresponding dispersion did not differ significantly from each other (Zedginidze, 2000).

For the qualitative assessment of the observed processes, we have developed the algorithm for calculating the characteristic parameter of the process $-\mathrm{S}$. determining the value of the criteria for different kind of deterministic processes. We compiled a program that performs sampling of the input signal and calculates the specified parameter as follows:

$$
S=\frac{1}{\mathrm{n}}\left[\sum_{\mathrm{i}=1}^{\mathrm{n}}\left|\left(\mathrm{x}_{\mathrm{i}}-127\right)\right|+\mathrm{B}_{1}+\mathrm{B}_{2}\right]
$$

where $x_{i}$-are the values of the quantized signal at a point $t_{i}$.

127 - is an average value of the signal in accordance with the code of analog-to-digital conversion, $\mathrm{n}$ number of observations, $B_{1}$ and $B_{2}$ - additional components whose values are determined by the frequency components, the upper UH and lower UL levels - respectively F1 and F2 values for the following expressions:

$$
\begin{aligned}
& B_{1 j(x j)}=\left\{\begin{array}{lr}
0, & x_{j}<U H \\
\left|x_{j}-127\right|, & x_{j} \geq U H
\end{array}\right. \\
& B_{1}=\sum\left|x_{j}-127\right|
\end{aligned}
$$

and similarly the lower level

$$
\begin{array}{cc}
B_{2 j(x k)}= \begin{cases}0, & x_{k}<\mathrm{UL} \\
\left|x_{k}-127\right|, & x_{k} \geq \mathrm{UL}\end{cases} \\
B_{2}=\sum\left|x_{k}-127\right| & i<k<n
\end{array}
$$

As a result, using expression (3) and (5) additional components $B_{1}$ and $B_{2}$ are calculated, that are shown in expression (1). The characteristic parameter of the process $-\mathrm{S}$ is also calculated.

Through repeated experiments, we get a data set of characteristic parameter $\mathrm{Si},(\mathrm{i}=1, \mathrm{~N})$, where $\mathrm{N}$ is the number of experiments conducted. On the basis of the resulting data set $\mathrm{Si}$, defined criteria for distinguishing values $\mathrm{kd}$. In addition, the program includes indicating index $\mathrm{m}$, taking only two values -0 and 1 . The value of indicating index depends on the criteria kd value. Provided that if $S$ $<\mathrm{kd}$, the process under study belongs to the accumulation process and the parameter of the indicator is zero $(\mathrm{m}=0)$, and if $\mathrm{S} \geq \mathrm{kd}$, the process under study belongs to the process of the release of energy (level) and the indicator index becomes equal to unity $(\mathrm{m}=1)$. Observing the values of $\mathrm{m}$ and counting the number of received pulses $\mathrm{Km}$ of the internal microcontroller counter in unit time $\mathrm{t}$, we receive quantitative assessment - power estimated parameter of generated output pulses $-\mathrm{P}=\mathrm{Km} / \mathrm{t}$.

Thus, the calculation of the characteristic parameter $\mathrm{S}$ and the determination of difference criterion value $\mathrm{kd}$, allowed to identify and distinguish the process accumulating (charge) power from the process releasing (discharge) energy. Observing the values of the indicator index $\mathrm{m}$ and counting the microcontroller internal counter produced pulses in a certain time t, let quantify the rate of power of the output pulses generated by analyzed device.

Conclusions. Thus, this approach has allowed to work out ways of finding a distinction criterion of different types of deterministic processes, allowing to identify and assess the quantitative index of the signals. For carried out experiments and analysis, was proved the correctness of the criterion of the differences between the two processes, and have been repeatedly received true results.

\section{REFERENCES}

1. Azmaiparashvili, Z., Poladashvili, M., \& Meskhidze, N. (2012). Georgia Patent No. P 6089.

2. AzmaiparaSvili, Z., Poodashvili, M., \& Meskhidze, N. (2015). Wireless power transmission devices. ENERGY: REGIONAL PROBLEMS AND DEVELOPMENT OPPORTUNITIES. Kutaisi.

3. Bluhm, H. (2006). Pulsed power systems. Principles and Applications. Berlin, Germany: Springer-Verlag Berlin Heidelberg.

4. Lyons, R. G. (2010). Understanding Digital Signal Processing. (3, Ed.) Pearson Education, 2010.

5. Zedginidze, I. (2000). Organization and Planning of Engineering Experiment. Tbilisi, Georgia: Georgian Technical University. 